\title{
La cinéradioscopie comme moyen d'analyse fonctionnelle après laryngectomie
}

\author{
Malthièry $\mathbf{E}^{\mathbf{1}}$, Letellier $\mathrm{P}^{\mathbf{2}}$, Gauvin $\mathrm{Y}^{\mathbf{2}}$, Predine-Hug $\mathrm{F}^{\mathbf{1}}$ \\ ${ }^{1}$ UFR d'Odontologie, Brest, France \\ ${ }^{2}$ Service d'ORL, CHU Morvan, Brest, France \\ eve.malthiery@chu-brest.fr
}

Les cancers du larynx sont des pathologies fréquentes souvent liées à une mauvaise hygiène de vie. Ils représentent environ $25 \%$ des cancers des voies aéro-digestives supérieures. Ils conduisent souvent à une intervention chirurgicale toujours mutilante. Après l'intervention, une exploration dynamique trouve tout son intérêt. La laryngectomie, qu'elle soit partielle ou totale, va entraîner des anomalies de la phonation et éventuellement de la déglutition (Stecewicz et al. 2006). La radioscopie permet d'objectiver le positionnement de la langue lors de la prononciation de certains phonèmes (Aramany et al. 1982) ainsi que de possibles dysfonctionnements des voies aéro-digestives supérieures, fausses routes, stagnation du bol alimentaire ...

Cette technique a été récemment appliquée chez deux patients avec un cancer du larynx traité par une intervention chirurgicale d'exérèse. Le premier cas concerne un patient de 58 ans, chez qui une pharyngo-laryngectomie totale a été effectuée pour un carcinome épidermoïde envahissant les trois étages du larynx. Le second cas est un patient de 82 ans, atteint d'un carcinome épidermoïde ayant amené à pratiquer une laryngectomie partielle sus-glottique, entraînant une diminution du volume et de la mobilité de la langue. La déglutition et la dynamique pharyngée ont été analysées. La radioscopie permet d'observer l'appui lingual lors de la prononciation de certains phonèmes, ainsi que la dynamique du carrefour oro-pharyngé. L'intervention chirurgicale lors de l'ablation d'une tumeur maligne n'est pas standard, elle dépend beaucoup de l'envahissement local du processus tumoral. L'emploi de la radioscopie dynamique se justifie alors tout particulièrement dans les suites d'interventions mutilantes. Elle permet d'établir un bilan fonctionnel post-opératoire.

Bien que cette technique comporte une irradiation importante, elle présente un intérêt, notamment dans les pathologies lourdes du larynx ou du pharynx nécessitant une exérèse mutilante, comme les laryngectomies. Elle permet alors de mettre en évidence la qualité fonctionnelle résiduelle des voies aéro-digestives supérieures. Elle est utile pour modeler une prothèse adaptée au volume et à la mobilité résiduelle de la langue (Davis et al. 1987). Elle permet également d'évaluer les bénéfices de la rééducation orthophonique (Logemann et al.1993). 